\title{
Destroying a near-extremal Kerr-Newman black hole
}

\author{
Alberto Saa ${ }^{1, *}$ and Raphael Santarelli ${ }^{2,0}$ \\ ${ }^{1}$ Departamento de Matemática Aplicada, UNICAMP, 13083-859 Campinas, SP, Brazil \\ ${ }^{2}$ Instituto de Física "Gleb Wataghin", UNICAMP, 13083-859 Campinas, SP, Brazil
}

\begin{abstract}
We revisit here a previous argument due to Wald showing the impossibility of turning an extremal Kerr-Newman black hole into a naked singularity by plunging test particles across the black hole event horizon. We extend Wald's analysis to the case of near-extremal black holes and show that it is indeed possible to destroy their event horizon, giving rise to naked singularities, by pushing test particles towards the black hole as, in fact, it has been demonstrated explicitly by several recent works. Our analysis allows us to go a step further and to determine the optimal values, in the sense of keeping to a minimum the backreaction effects, of the test particle electrical charge and angular momentum necessary to destroy a given near-extremal Kerr-Newman black hole. We describe briefly a possible realistic scenario for the creation of a Kerr naked singularity from some recently discovered candidates to be rapidly rotating black holes in radio galaxies.
\end{abstract}

PACS numbers: 04.20.Dw, 04.70.Bw, 97.60.Lf

\section{INTRODUCTION}

There has been recently a revival of interest in the problem of turning a black hole into a naked singularity by means of classical and quantum processes, see, for instance, 1] and [2] for references and a brief review with a historical perspective. Such a problem is intimately related to the weak cosmic censorship conjecture 3 , [4]. Indeed, the typical facility in covering a naked singularity with an event horizon and the apparent impossibility of destroying a black hole horizon [5] have strongly endorsed the validity of the conjecture along the years, albeit it has started to be challenged recently.

The most generic asymptotically flat black hole solution of Einstein equations we can consider is the KerrNewman black hole, which is completely characterized by its mass $M$, electric charge $Q$ and angular momentum $J=a M$. The distinctive feature of a black hole, namely the existence of an event horizon covering the central singularity, requires

$$
M^{2} \geq a^{2}+Q^{2}
$$

with the equality corresponding to the so-called extremal case. If (1) does not hold, the central singularity is exposed, giving rise to a naked spacetime singularity, which should not exist in Nature according to the weak cosmic censorship conjecture. All the classical results on the impossibility of destroying black hole horizons were obtained by considering extremal black holes. The first work arguing that it would be indeed possible to destroy the horizon of a near-extremal black hole is quite recent and it was due to Hubeny [6], which considered a Reissner-Nordström $(a=0)$ black hole. The physical possibility of destroying the horizon of a near-extremal

*Electronic address: asaa@ime.unicamp.br

†Electronic address: telsanta@ifi.unicamp.br black hole by over-spinning or over-charging it with the absorption of test particles or fields is nowadays a very active field of research and debate [1, 2, 7 -16].

The impossibility of destroying the event horizon by plunging test particles into an extremal Kerr-Newman black hole is clear and elegantly summarized in Wald argument [17], which we briefly reproduce here. We consider test particles with energy $E$, electric charge $e$, and orbital angular momentum $L$. The test particle approximation requires $E / M \ll 1, L / a M \ll 1$, and $e / Q \ll 1$, assuring, in this way, that backreaction effects are negligible. The particle angular momentum $L$ is assumed to be aligned with the black hole angular momentum $J$, and both $Q$ and $e$ are assumed, without loss of generality, to be positive. According to the laws of black hole thermodynamics (see, for instance, section 33.8 of [18]), after the capture of a test particle, the black hole will have total angular momentum $a M+L$, charge $Q+e$ and mass no greater than $M+E$. In order to form a naked singularity, one needs

$$
(M+E)^{2}<\left(\frac{a M+L}{M+E}\right)^{2}+(e+Q)^{2},
$$

which implies, for extremal black holes and in the test particle approximation,

$$
E<\frac{Q e M+a L}{M^{2}+a^{2}} .
$$

However, in order to assure that the test particle be indeed plunged into the black hole, its energy must obey [18]

$$
E \geq E_{\min }=\frac{Q e r_{+}+a L}{r_{+}^{2}+a^{2}},
$$

with

$$
r_{+}=M+\sqrt{M^{2}-a^{2}-Q^{2}}
$$

being the event horizon radius of the black hole. For an extremal black hole, $r_{+}=M$, and it is clear that (3) and 
(4) will not be fulfilled simultaneously, implying that one cannot turn an extremal Kerr-Newman black hole into a naked singularity by plunging test particle across its event horizon. Wald presents also a similar argument for the case of dropping spinning uncharged particles into a Kerr $(Q=0)$ black hole. Notwithstanding, de Felice and Yunqiang [19] showed that it would be indeed possible to transform a Reissner-Nordström black hole in a KerrNewman naked singularity after capturing an electrically neutral spinning body.

The purpose of this work is to extend Wald's original analysis [17] to the case of near-extremal Kerr-Newman black holes and show explicitly that it is indeed possible to over-spin and/or over-charge near-extremal black holes by plunging test particle across their event horizon while keeping backreaction effects to a minimum. All the recently proposed mechanisms to destroy a near-extremal black-hole by using infalling test particles are accommodated in our analysis. Furthermore, we determine the optimal values, in the sense that they keep backreaction effects to a minimum, of the electrical charge and angular momentum of the incident test particle in order to destroy a near-extremal Kerr-Newman black hole with given mass, charge, and angular momentum. We show also that it is not strictly necessary to plunge the particles across the black hole horizon, but they can be thrown from infinity and proceed towards to the black hole following a geodesics, minimizing in this way any back reaction effect associated with the specific mechanism to release the particle near, os push it against, the black hole horizon. As an explicit example, we consider some recently discovered candidates to be rapidly rotating black holes in radio galaxies and show how it would be possible to create Kerr naked singularities from them with minimal backreaction effects.

\section{NEAR-EXTREMAL KERR-NEWMAN BLACK HOLES}

We call near-extremal a Kerr-Newman black hole for which

$$
\delta^{2}=M^{2}-a^{2}-Q^{2}>0, \quad \frac{\delta}{M} \ll 1 .
$$

For a near-extremal black hole, the condition (1) for the creation of a naked singularity by absorbing a test particle with energy $E$, electric charge $e$, and orbital angular momentum $L$ implies that

$$
E<E_{\max }=\frac{Q e M+a L}{M^{2}+a^{2}}-\frac{M^{3}}{2\left(M^{2}+a^{2}\right)}\left(\frac{\delta}{M}\right)^{2} .
$$

It is more convenient here to introduce a parametrization for near-extremal black holes

$$
\begin{aligned}
a & =\sqrt{M^{2}-\delta^{2}} \cos \alpha, \\
Q & =\sqrt{M^{2}-\delta^{2}} \sin \alpha,
\end{aligned}
$$

with $0 \leq \alpha \leq \pi / 2$. In this way, near-extremal black holes are characterized by the triple $(M, \delta, \alpha)$. For instance, near-extremal Kerr and Reissner-Nordström black holes correspond, respectively, to $(M, \delta, 0)$ and $(M, \delta, \pi / 2)$. After some straightforward algebra, it is possible to show that

$$
E_{\max }=A-\frac{M+A \sin ^{2} \alpha}{2+2 \cos ^{2} \alpha}\left(\frac{\delta}{M}\right)^{2}
$$

where only terms up to second order in $(\delta / M)$ were kept, and

$$
A=\frac{(L / M) \cos \alpha+e \sin \alpha}{1+\cos ^{2} \alpha} \geq 0 .
$$

The event horizon for near-extremal black holes are located at $r_{+}=M+\delta$, which implies that the condition (14) assuring that the particle is to be captured reads

$E \geq E_{\min }=A-B\left(\frac{\delta}{M}\right)-\left(\frac{\left(2+\sin ^{2} \alpha\right) A-4 B}{2+2 \cos ^{2} \alpha}\right)\left(\frac{\delta}{M}\right)^{2}$

where, again, only terms up to $(\delta / M)^{2}$ were kept and

$$
B=\frac{2(L / M) \cos \alpha+e \sin ^{3} \alpha}{\left(1+\cos ^{2} \alpha\right)^{2}} \geq 0 .
$$

It is clear that for the extremal case $(\delta=0)$ we have Wald's result $E_{\max }=E_{\min }$, implying that (7) and (12) cannot be fulfilled simultaneously. However, for $\delta>0$ it is indeed possible to a test particle obey (7) and (12). The intersection of $E_{\max }$ and $E_{\min }$ in the $(\lambda, \varepsilon)$ plane corresponds to the straight line

$$
2 \lambda \cos \alpha+\varepsilon \sin ^{3} \alpha=\frac{1+\cos ^{2} \alpha}{2},
$$

where $\lambda=L / M \delta$ and $\varepsilon=e / \delta$. The forbidden region, where no naked singularity is formed, is depicted in Fig. 1.

\section{A. Optimal test particles}

In all derivations done so far, we have used the test particle approximation. The idea of minimizing the values of $E, L$, and $e$ necessary to destroy the black hole is more than a simple requirement of consistence. It helps to assure that back reaction effects are negligible and, consequently, that it will not be possible to restore the black hole event horizon by means of any subdominant physical process. The first, and maybe the more natural, criterium of optimality we can devise here is to require minimal test particle total energy $E$, which is given by $E_{\text {min }}$ in (12). The test particle minimal energy $E(\lambda, \varepsilon)$ necessary to destroy the black hole corresponds to the minimum value of $E_{\text {min }}$, subject to the restriction (14). This is a simple linear optimization problem [20], and the solution is known to correspond to one of the points $(0, \varepsilon)$ 


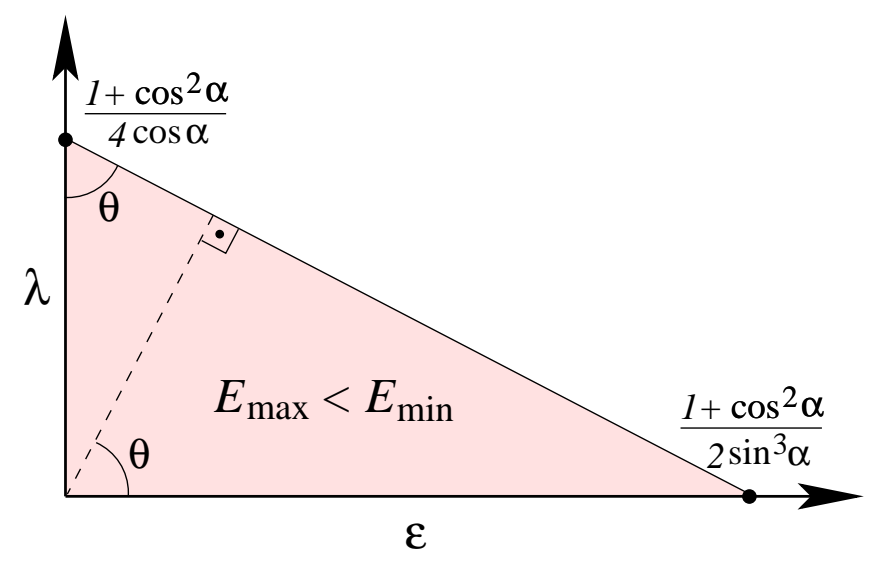

FIG. 1: In the shadowed region, no naked singularity is formed. Conditions (7) and (12) are simultaneously fulfilled in the region above the straight line in the $(\lambda, \varepsilon)$ plane, where $\lambda=L / M \delta$ and $\varepsilon=e / \delta$. The validity of the test particle approximation, of course, does not allow arbitrary large values of $\lambda$ and $\varepsilon$. Hence, the allowed region for the creation of naked singularities is delimited by the straight line and by the validity of the test particle approximation.

or $(\lambda, 0)$, i.e., the minimal energy $E$ will be given either by

$$
E(0, \varepsilon)=\frac{\delta}{2 \sin ^{2} \alpha}
$$

or

$$
E(\lambda, 0)=\frac{\delta}{4}
$$

It is clear that $E(\lambda, 0)<E(0, \varepsilon)$ for any value of $\alpha$, suggesting that the best option to turn a black hole into a naked singularity would be to plunge an uncharged particle, irrespective of the value of $\alpha$. However, we see from (14) that $\lambda$ can increase considerably for small $\alpha$, despite of $E(\lambda, 0)$ being a minimum. The minimization of $E(\lambda, \varepsilon)$ does not guarantee the minimization of $L$ and $e$, risking the validity of the test particle approximation. In order to avoid theses problems, we will require that $\lambda^{2}+\varepsilon^{2}$ be minimal for optimal test particles. Such a requirement corresponds to select the nearest point of the straight line (14) to the origin in the $(\lambda, \varepsilon)$ plane. From Fig. 1 and simple trigonometry, we have that a test particle with angular momentum $L$ and electrical charge $e$ obeying

$$
\frac{e M}{L}=\frac{\sin ^{3} \alpha}{2 \cos \alpha}=\frac{(Q / a)^{3}}{2+2(Q / a)^{2}}
$$

is the optimal test particle to turn a near-extremal KerrNewman black with parameters $(M, \delta, \alpha)$ into a naked singularity. The energy associated with the optimal parameters (17) is in the range delimited by (15) and (16).

It is clear from (17) that the optimal test particle to turn a near-extremal Kerr black hole $(\alpha=0)$ into a naked singularity should be electrically neutral $(e=0)$. Moreover, from Fig. 1] we see that the particle must have
$L / M \geq \delta / 2$. The minimum particle energy is given by (16), assuring the validity of the test particle approximation in this case $(E / M \ll 1$ and $L / a M \ll 1)$ provided the black hole be near-extremal $(\delta / M \ll 1)$. For the case of a Reissner-Nordström black hole $(\alpha=\pi / 2)$, the optimal test particle must be charged, with $e>\delta / 2$, and have vanishing orbital angular momentum $(L=0)$. The minimum particle energy for this case is given by $E=\delta / 2$, according to (15). The validity of the test particle approximation and the minimization of any backreaction effect is assured also in this case.

\section{THROWING PARTICLES FROM INFINITY}

The expression for $E_{\min }$ given by equation (41) corresponds to the minimal energy that a test particle can have at the black hole horizon. On the other hand, the minimal energy that a particle can have anywhere on the equatorial plane outside a Kerr-Newman black hole is given by the effective potential [18]

$$
V(r)=\frac{\beta+\sqrt{\beta^{2}-\nu \gamma_{0}}}{\nu},
$$

where

$$
\begin{aligned}
\beta & =(L a+Q e r)\left(r^{2}+a^{2}\right)-L a \Delta, \\
\nu & =\left(r^{2}+a^{2}\right)^{2}-a^{2} \Delta, \\
\gamma_{0} & =(L a+Q e r)^{2}-\left(L^{2}+\mu^{2} r^{2}\right) \Delta,
\end{aligned}
$$

with $\mu$ being the particle rest mass and

$$
\Delta=r^{2}-2 M r+a^{2}+Q^{2} .
$$

The event horizon $r_{+}$is the outermost zero of $\Delta$ and equation (4) corresponds to the potential (18) evaluated on $r=r_{+}$. For $r \rightarrow \infty$ one has $V \rightarrow \mu$, as it is expected for any asymptotically flat solution. For a particle of energy $E$, the points for which $V(r)=E$ are return points and delimit the classically allowable region for the particle motion. In order to assure that a particle with energy $E$ thrown from infinite reaches the horizon, we need to have $E>V(r)$ in the exterior region of the black hole. In some cases, one can have that $\mu>E_{\min }$, i.e., the energy necessary for the incident test particle reach the horizon is smaller than its rest mass. This is not a surprise in gravitational systems, but, of course, this trajectory cannot start from infinity. To follow this trajectory, a particle must be released near the horizon, by some external mechanism. This is an extra and unnecessary complication in our analysis. The external mechanism could be subject to some backreaction or subdominant physical effect that could eventually prevent the particle of entering the black hole. This can be avoided if we adjust the particle rest mass $\mu$ properly. An explicit example can enlighten this point.

Let us consider the case of a Kerr black hole $(Q=$ $0)$. (For a recent review on equatorial orbits in Kerr 


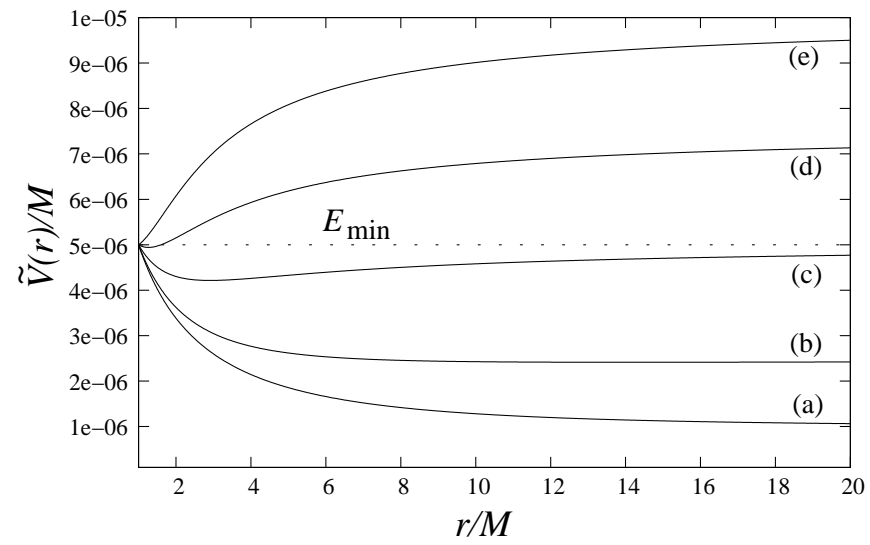

FIG. 2: The effective potential (18) for a test particle with angular momentum $L$ and rest mass $\mu$ around an extremal Kerr black hole with mass $M$. For this figure, $L / M^{2}=10^{-5}$ and $\mu / M=10^{-6}(\mathrm{a}), 2.5 \times 10^{-6}(\mathrm{~b}), 5 \times 10^{-6}(\mathrm{c}), 7.5 \times 10^{-6}(\mathrm{~d})$, and $10^{-5}(\mathrm{e})$. The doted horizontal line corresponds to the value of the effective potential at the black hole horizon $\left(E_{\min }\right)$, which does not dependent on $\mu$.

black holes and naked singularities, see 21].) For a nearextremal Kerr black hole, the effective potential (18) can be written as

$$
V(r)=\tilde{V}(r)+O\left((\delta / M)^{2}\right),
$$

where $\tilde{V}(r)$ stands for the effective potential for the extremal Kerr black hole, which can be calculated from (18) with $Q=0$ and $a=M$. Fig. 2 depicts the effective potential $\tilde{V}(r)$ for different values of $\mu$. Typically, the choice of $\mu<E_{\min }$ will allow the particle to reach the horizon when thrown from infinite with energy $E \approx E_{\min }$.

\section{FINAL REMARKS}

We have shown that the Wald analysis [17] can be extended to the case of near-extremal Kerr-Newman black holes, allowing the accommodation of the recent proposals to over-spin or over-charge near-extremal black holes in a single and simpler framework. Moreover, we could determine the optimal parameters for a test particle in order to destroy a black hole while keeping backreaction effects to a minimum. An explicit and realistic example here will be valuable to enlighten these points.
There are some evidences of rapidly rotating black holes in quasars [22] and radio galaxies 23]. These black holes are very massive, having typically $M \approx 10^{8} M_{\odot}$, and can attain an angular momentum such that $a / M \gtrsim$ 0.9. Let us suppose we have one of these black holes with $\delta / M \approx 10^{-5}$. According to Section 2, the capture of a test body with $L / M^{2}=10^{-5}$ will suffice to the creation of a naked singularity. For this case, $E_{\min } \approx 10^{3} M_{\odot}$. A test body with mass comparable to the moon mass, $\mu \approx 4 \times 10^{-8} M_{\odot}$, will certainly be able to reach the horizon if thrown from infinity with angular momentum $L / M^{2}=10^{-5}$ (See Fig. 2). The minimal necessary energy for a test body reach the horizon with such orbital angular momentum is given by (12), $E_{\min } / M=10^{-5}\left(1-10^{-5}\right) / 2$. On the other hand, with this angular momentum, any test body captured with energy $E / M<E_{\max } / M=10^{-5}\left(1-10^{-5} / 2\right) / 2$ will destroy the black hole. Hence, any test body thrown from infinity with angular momentum $L / M^{2}=10^{-5}$ and energy $E$ such that $E_{\min }<E<E_{\max }$ will produce a naked singularity. Furthermore, the validity of the test particle approximation is assured in this example. It is important also to notice that the horizon radius of such rapidly rotation black holes are of the order of $10^{8} \mathrm{~km}$, very large when compared with the moon radius of $1.7 \times 10^{3} \mathrm{~km}$. A body with mass and size comparable the the moon would be well described by the test particle approximation even when crossing the horizon of such rapidly rotating black holes. Indeed, it is very hard to devise any backreaction effect that could prevent the formation of a naked singularity in this case. We close noticing that, probably, the Thorne limit $a / M \approx 0.998$ [24] corresponds to the most realistic near-extremal astrophysical Kerr black hole. For such case, $\delta / M \approx 6 \%$ and we have analogous results to the preceding example. However, in this case, the validity of the test particle approximation could be questioned and we could have appreciable backreaction effects.

\section{Acknowledgments}

This work was supported by FAPESP and CNPq. The authors wish to thank V.E. Hubeny for enlightening conversations.
[1] G. E. A. Matsas and A. R. R. da Silva, Phys. Rev. Lett. 99, 181301 (2007) arXiv:0706.3198 [gr-qc]].

[2] T. Jacobson and T. P. Sotiriou, J. Phys. Conf. Ser. 222, 012041 (2010) arXiv:1006.1764 [gr-qc]].

[3] R. M. Wald, arXiv:gr-qc/9710068.

[4] C. J. S. Clarke, Class. Quantum Grav. 10, 1375 (1994).

[5] J. M. Cohen and R. Gautreau, Phys. Rev. D 19, 2273
(1979).

[6] V. E. Hubeny, Phys. Rev. D 59, 064013 (1999) arXiv:gr-qc/9808043.

[7] M. Richartz and A. Saa, Phys. Rev. D 78, 081503 (2008) arXiv:0804.3921 [gr-qc]].

[8] S. Hod, Phys. Rev. Lett. 100, 121101 (2008) arXiv:0805.3873 [gr-qc]]; Phys. Lett. B 668, 346 (2008) 
arXiv:0810.0079 [gr-qc]].

[9] G. E. A. Matsas, M. Richartz, A. Saa, A. R. R. da Silva and D. A. T. Vanzella, Phys. Rev. D 79, 101502 (2009) arXiv:0905.1077 [gr-qc]].

[10] T. Jacobson and T. P. Sotiriou, Phys. Rev. Lett. 103, 141101 (2009) [Erratum-ibid. 103, 209903 (2009)] arXiv:0907.4146 [gr-qc]].

[11] E. Berti, V. Cardoso, L. Gualtieri, F. Pretorius and U. Sperhake, Phys. Rev. Lett. 103, 239001 (2009) arXiv:0911.2243 [gr-qc]].

[12] M. Bouhmadi-Lopez, V. Cardoso, A. Nerozzi and J. V. Rocha, Phys. Rev. D 81, 084051 (2010) arXiv:1003.4295 [gr-qc]].

[13] T. Jacobson and T. P. Sotiriou, arXiv:1006.1763 [gr-qc].

[14] G. Chirco, S. Liberati and T. P. Sotiriou, Phys. Rev. D 82, 104015 (2010) arXiv:1006.3655 [gr-qc]].

[15] E. Barausse, V. Cardoso and G. Khanna, Phys. Rev.
Lett. 105, 261102 (2010) arXiv:1008.5159 [gr-qc]].

[16] J. V. Rocha and V. Cardoso, arXiv:1102.4352 [gr-qc]

[17] R. Wald, Ann. Phys. 82, 548 (1974).

[18] C.W. Misner, K.S. Thorne, and J.A. Wheeler, Gravitation, W.H. Freeman, San Francisco (1973).

[19] F. de Felice and Y. Q. Yu, Class. Quant. Grav. 18, 1235 (2001).

[20] G.B. Danzig and M.N. Thapa, Linear Programming 2: Theory and Extensions, Springer, Berlin (2003).

[21] D. Pugliese, H. Quevedo and R. Ruffini, arXiv:1105.2959 [gr-qc].

[22] J. M. Wang, Y. M. Chen, L. C. Ho and R. J. McLure, Astrophys. J. 642, L111 (2006) arXiv:astro-ph/0603813.

[23] Q. Wu, X. Cao and D. X. Wang, arXiv:1104.3235] [astroph.HE].

[24] K.S. Thorne, ApJ. 191, 507 (1974). 